\title{
A Numerical Model for Low-Frequency Equatorial Dynamics
}

\author{
MARK A. CANE ${ }^{1}$ and RANDAll J. PATtON ${ }^{2}$ \\ Department of Meteorology and Physical Oceanography, M.I.T., Cambridge, MA 02139
}

(Manuscript received 27 August 1983, in final form 28 September 1984)

\begin{abstract}
A fast, efficient numerical procedure for modeling the linear low-frequency motions on an equatorial beta plane is developed. The model is capable of simulating the seasonal and interannual variability in realistically shaped ocean basins forced by realistic winds. The timestep allowed is the order of days rather than hours as allowed by more conventional schemes. The numerical method is designed around the special characteristics of low-frequency equatorial waves. A crucial element is the formulation of proper boundary conditions, including those for a partial boundary such as the western end of the Gulf of Guinea.

The response of an Atlantic-shaped basin to a periodic wind is compared with the analytic results of Cane and Sarachik for a meridionally unbounded ocean. The response along the equator is essentially the same. A narrow boundary layer forms along the Guinea coast, too narrow to be a single coastally trapped wave: it is the sum of many modes. The near lack of phase variation along the coast and the smallness of the phase difference between the equator and the coast are additional contrasts to the results for an initial value problem studied by Moore and others. The implication is that the annually recurring upwelling in the Gulf of Guinea cannot be adequately modeled as the response to an impulsively applied wind stress.
\end{abstract}

\section{Introduction}

Beginning with the seminal work of Lighthill (1969), linear shallow water models have proven remarkably useful for explaining equatorial phenomena on seasonal and interannual time scales. While it is true that most of the success of these models comes in comparing relatively unstructured, integrated quantities like dynamic topography, it is nonetheless unexpected that theories that invoke only such simple physics should work so well. (For a list of recent examples see Cane and Sarachik, 1983.) Most of the work along these lines has used simple geometry (e.g., meridionally infinite equatorial beta plane) and highly idealized wind forcings, but recently a number of studies with realistic basin shapes and wind forcings have been carried out (e.g., Busalacchi and O'Brien, 1980,1981 ). One of the key points of the present work is that such realistic studies are essential if the true applicability of shallow water theory is to be tested: results from highly idealized calculations (e.g., initial value problems with switched on winds; featureless wind systems) are often misleading.

Even the studies we refer to as realistic have been highly idealized. They neglect nonlinearity, heat exchange with the atmosphere, surface layer mixing and most of the complexity of the thermal stratifica-

\footnotetext{
${ }^{1}$ Present affiliation: Lamont-Doherty Geological Observatory, Palisades, NY 10964.

${ }^{2}$ Present affiliation: Dynamics Technology, 22939 Hawthorne Blvd., Torrance, CA 90505.
}

tion of the real oceans. Of course, these simplifications have the virtue of making the results more easily understood. Even so, interpretations are made in terms of only a subset of the possible motions that the shallow water system allows: the low-frequency, long zonal-scale equatorial Kelvin and Rossby waves. While these are the only motions relevant in such problems, the shallow water equations also describe inertia-gravity waves with much higher frequencies. Their presence forces the numerical models employed in the realistic calculations to use time steps that are very small compared with the time scales of interest (i.e., a few hours compared with a few months.)

In the present study we develop a numerical model that filters all but the low frequency, long zonal-scale waves. The physically interesting motions are retained, but a very long timestep (we use 10 days) is allowed. This makes the model extremely fast, so that even with modest computing resources many calculations may be carried out. It is then possible, for example, to run dozens of simulations in order to explore what features of the wind system are responsible for such oceanic responses as the upwelling in the Gulf of Guinea (cf. Patton, 1981). It is also feasible to perform many experiments with a stratified ocean model by decomposing the response into a sum of vertical modes (e.g., Cane, 1984).

Our numerical procedure has been designed with the characteristics of the physics of the system in mind. Our approach is based on the extensive theory for the shallow water system developed by Cane and Sarachik (1976, 1977, 1979, 1981; henceforth CSI, 
CSII, CSIII, CSIV, respectively). We particularly exploit the fact that of the allowed free motions, all propagate westward with the exception of the equatorial Kelvin wave. It is essential to our procedure to extract the Kelvin component of the solution. We do so by projecting the Kelvin mode onto the governing equations; this is possible because the vector shallow water modes $(u, v, h)$ form an orthogonal and complete set. (The proof of this for a meridionally bounded basin is given in CSIII, Appendix A.)

It is a relatively simple matter to find the equations that govern the low-frequency, long-wave motions (cf. Section 2), but the formulation of proper initial and boundary conditions is far less obvious. First, the restricted class of motions remaining after the equations are filtered do not satisfy the obvious boundary conditions of no normal flow. Second, the usual algorithms for calculating boundary conditions (e.g., Moore and Philander, 1977) are based on a complete decomposition into normal modes, something we wish to avoid as computationally extravagant. Moreover, they have no obvious extension to a meridionally bounded ocean. Here we construct proper, computationally efficient boundary conditions based on the results presented in CSIII. Section 2 of this paper reviews the equations for our physical model, including initial and boundary conditions. It is obviously desirable to be able to model a more realistic basin geometry than a simple rectangle. A complete extension of the CS theory to partial boundaries was developed in DuPenhoat et al. (1983, hereafter DCP). In Section 3, we present the results needed to treat a partial boundary such as the Gulf of Guinea, the case to be considered in this paper.

Before formulating the present model we considered the more obvious alternative of a special procedure in which the solution would be represented as a sum of the normal modes of the shallow water equations on an equatorial beta plane. For low frequencies these are the Kelvin wave and Rossby waves. For realistic problems this alternative has (at least) three serious drawbacks:

(i) the nonexistence of a "fast Hermite transform" means it is slow and awkward to obtain the needed projections of the forcing fields. This difficulty is exacerbated in a meridionally bounded basin where the eigenfunctions are sums of parabolic cylinder functions which themselves must be obtained numerically (cf. CSIII).

(ii) At a partial boundary such as the western end of the Gulf of Guinea, the meridional structure of the eigenfunctions changes. Hence continuing the integration across such a line requires that one set of eigenfunctions be expanded in terms of the other.

(iii) The eigenfunctions have poor convergence properties. For example, the eastern boundary reflection of a low frequency Kelvin wave has meridionally uniform height at the coast. It takes very many Hermite functions to represent a constant height out to, say, $15^{\circ} \mathrm{N}$.

Section 4 describes our numerical scheme. We decompose the solution into (i) a Kelvin mode and (ii) everything else (Rossby modes and an anti-Kelvin mode). The Kelvin mode is integrated analytically, eastward along characteristics. Everything else is marched collectively westward using an implicit scheme that is centered second-order in time and space. Marching westward obviates the need to invert a large matrix; only a simple tridiagonal system must be solved at each longitude. Thus the scheme is computationally efficient and the time step is limited by accuracy considerations, rather than stability criteria.

In this paper we use the model to extend the purely analytical results of CSIV on the baroclinic response of equatorial oceans to periodic forcing to the analytically intractable case of a basin resembling the Atlantic in having a feature like the Gulf of Guinea. Section 5 treats a wind forcing identical to that in CSIV, while Section 6 considers the case where the forcing is confined to the western part of the ocean (cf. Moore et al., 1978). Some implications of our results for theories for upwelling in the Gulf of Guinea are pointed out. The final section summarizes our results.

\section{The physical model}

We begin from the linear shallow water equations on an equatorial beta plane:

$$
\left.\begin{array}{l}
u_{t}-y v+h_{x}=F-r u \\
v_{t}+y u+h_{y}=G-r v \\
h_{t}+u_{x}+v_{y}=Q-r h
\end{array}\right\} .
$$

The equations have been nondimensionalized in the usual equatorial way: the time scale is $T=(c \beta)^{-1 / 2}$ and lengthscale is $L=(c / \beta)^{1 / 2}$, where $c=(g H)^{1 / 2}$ is a scale for wave speeds. Here $H$ may be interpreted as the equivalent depth of a particular vertical mode or as $H=\Delta \rho /\left(\rho H^{\prime}\right)$ for a reduced gravity model with an active layer of mean depth $H^{\prime}$. The external forcings $F, G$, and $Q$, are due to zonal wind stress, meridional wind stress and buoyancy flux, respectively. A Rayleigh friction damping with decay time $r^{-1}$ has been included; note that, with the definitions

$\left(u^{*}, v^{*}, h^{*}, F^{*}, G^{*}, Q^{*}\right)=e^{r t}(u, v, h, F, G, Q)$,

the equations for the starred variables are formally the same as (2.1) with $r=0$. Our numerical model treats friction in this way; in the remainder of this section we will assume $r=0$. The appropriate initial 
and boundary conditions for the system (2.1) in a rectangular basin $0 \leqslant x \leqslant x_{E}, y_{S} \leqslant y \leqslant y_{N}$ are

$$
\begin{gathered}
u=v=h=0 \quad \text { at } t=0, \\
\mathbf{u} \cdot \mathbf{n}=0 \quad \text { at } x=0, x_{E} ; \mathrm{y}=\mathrm{y}_{S}, y_{N}
\end{gathered}
$$

when $\mathbf{n}$ is normal to the wall. The unforced solutions to (2.1) may be classified into five types (CSIII):

1) Equatorial Kelvin waves, propagating energy eastward with $v=0$ and $u$ and $h$ equal and proportional to

with

$$
\psi_{-1}(y)=C^{-1} e^{-y^{2} / 2}
$$

$$
C^{2}=\int_{y_{S}}^{y_{N}} e^{-y^{2}} d y
$$

2) Long Rossby waves propagating energy westward;

3) An "anti-Kelvin wave," related to coastal Kelvin waves, propagating energy westward;

4) Short Rossby waves, propagating energy slowly eastward;

5) Inertia-gravity waves.

Since we are interested in seasonal responses and large-scale features of the wind field, we make the low-frequency, long zonal-scale approximation:

$$
\frac{\partial}{\partial t}=\frac{\partial}{\partial x}=\mathrm{O}(\epsilon), \quad \epsilon \ll 1
$$

Scaling (2.1) accordingly reveals that $v=\mathrm{O}(\epsilon u), h$ $=\mathrm{O}(u)$ and

$$
\begin{aligned}
u_{t}-y v+h_{x} & =F, \\
y u+h_{y} & =G+\mathrm{O}\left(\epsilon^{2}\right), \\
h_{t}+u_{x}+v_{y} & =Q .
\end{aligned}
$$

We make no assumption about the relative magnitude of $F$ and $G$ but we note that if they are of the same order dimensionally, then $G$ is $O(\epsilon)$ relative to the left-hand side of $(2.5 \mathrm{~b})$.

The free solutions to the system (2.5) are only of types 1 ), 2), and 3 ), with the long Rossby waves 2 ) modified to be nondispersive. The high frequency inertia-gravity waves 5) and the short wavelength Rossby waves 4) have been eliminated. As a consequence, the initial conditions (2.3) and boundary conditions (2.4) can no longer be satisfied. As shown in CSI and CSII the appropriate initial conditions are now

$$
\begin{gathered}
u=-y \tilde{L}^{-1}(G) ; \quad h=\frac{\partial}{\partial y} \tilde{L}^{-1}(G) ; \\
v=\tilde{L}^{-1}(y F+\partial Q / \partial y),
\end{gathered}
$$

where $\tilde{L} \equiv \partial^{2} / \partial y^{2}-y^{2}$ and the boundary conditions are

$$
\begin{gathered}
v=0 \quad \text { at } y=y_{S}, y_{N}, \\
u=0 \text { at } x=x_{E}, \\
\int_{y_{S}}^{y_{N}} u d y=0 \text { at } x=0
\end{gathered}
$$

(on the last of these also see CSIV, p. 657).

In CSIII, it was shown that the wave solutions to (2.1) or (2.5) form a complete and orthogonal set. We will make extensive use of the fact that the Kelvin mode is orthogonal to all other modes: the solution to (2.5) may be written as

$$
\begin{aligned}
(u, v, h)= & a_{K}(x, t)\left[\psi_{-1}(y), 0, \psi_{-1}(y)\right] \\
& +\left[u^{\prime}(x, y, t), v^{\prime}(x, y, t), h^{\prime}(x, y, t)\right],
\end{aligned}
$$

where the prime quantities are long Rossby (type 2 ) and anti-Kelvin (type 3 ) modes. Therefore,

$\left(\psi_{-1}, 0, \psi_{-1}\right) \cdot\left(u^{\prime}, v^{\prime}, h^{\prime}\right)$

$$
=\int_{y_{S}}^{y_{N}} \psi_{-1}\left(u^{\prime}+h^{\prime}\right) d y=0 .
$$

In terms of the decomposition (2.5) into a Kelvin mode and all other modes, (2.9) may be expressed as

$a_{K}(x=0, t) \int_{y_{S}}^{y_{N}} \psi_{-1}(y)$

$$
=-\int_{y_{S}}^{y_{N}} u^{\prime}(x=0, y, t)
$$

and $(2.8)$ as

$$
u^{\prime}\left(x=x_{E}, y, t\right)=-a_{K}\left(x=x_{E}, t\right) \psi_{-1}(y) .
$$

In addition (2.8) together with (2.5b) determines that

$$
h\left(x=x_{E}, y, t\right)=h_{0}(t)+\int_{0}^{y} G\left(x=x_{E}, y, t\right) d y .
$$

Hence at $x=x_{E}$,

$$
h^{\prime}=h_{0}+\int_{0}^{y} G d y-a_{K} \psi_{-1}
$$

The integration constant $h_{0}(t)$ can be found in terms of $a_{K}$ by projecting the Kelvin wave onto this expression for $h$ together with $u=0$ [as per (2.8)]; the result is (cf. CSIII, p. 379)

$$
\begin{aligned}
h_{0}=\left\{a_{K}-\int_{y_{S}}^{y_{N}} d y \psi_{-1}(y) \int_{0}^{y} G\left(y^{\prime}\right) d y^{\prime}\right\} \\
\times\left[\int_{y_{S}}^{y_{N}} \psi_{-1}(y) d y\right]^{-1} .
\end{aligned}
$$

\section{Boundary conditions for the Gulf of Guinea}

In order to represent the geometry of the Gulf of Guinea we cut a corner north of $y=b$ and east of $x$ $=x_{B}$ out of the ocean basin (Fig. 1). The boundary conditions at the new solid boundaries are again that 
the normal velocity vanishes, but the influence of the partial boundary on propagating planetary waves is not obvious.

First consider a low frequency equatorial Kelvin wave arriving at longitude $x_{B}$ from the west with unit amplitude [i.e., $u=h=\psi_{-1}(y)$; cf. (2.4)]. When the Kelvin wave encounters the boundary, part of the wave will be reflected as long Rossby waves $\left(u_{r}, v_{r}\right.$, $h_{r}$ ), as is the case at a full boundary, but south of $b$ and east of $x_{B}$, there also will be a transmitted Kelvin wave. One might expect the Kelvin wave to pass through unchanged, so that its transmitted amplitude is also one. The reflected Rossby waves would then be absent south of $y=b$ since $u$ and $h$ must be continuous across $x_{B}$. In general, however, these conditions are inconsistent with geostrophy $(2.5 \mathrm{~b})$ and the fact that no new Kelvin waves can exist west of $x_{B}$.

Our method for calculating the influence of the partial boundary is similar to that used by Cane and DuPenhoat (1982) to find the influence of islands on equatorial waves. It has been described in detail in DuPenhoat $e t$ al. (1983), henceforth referred to as DCP. We summarize the results here.

For the problem described above of the incident Kelvin wave of unit amplitude, the amplitude $T^{K}$ of the transmitted Kelvin wave is

$$
T^{K}=2 /\left[2 \int_{y_{S}}^{b} \psi_{-1}^{2} d y+\psi_{-1}(b) \int_{b}^{y_{N}} \psi_{-1} d y\right]
$$

while at $x=x_{B}$ the reflected Rossby waves take the form

$$
\begin{gathered}
u^{r}=-\psi_{-1}(y) ; h^{r}=D^{K}-\psi_{-1}(y) \text { for } y \geqslant b, \\
u^{r}=h^{r}=\left(T^{K}-1\right) \psi_{-1}(y) \text { for } y<b
\end{gathered}
$$

where $D^{K}$, the height of the total solution along the boundary north of $b$, is

$$
D^{K}=T^{K} \psi_{-1}(b)
$$

The other situation to be considered is Rossby (and anti-Kelvin) waves propagating in from the east and encountering the corner at $x_{B}$. Say their amplitude

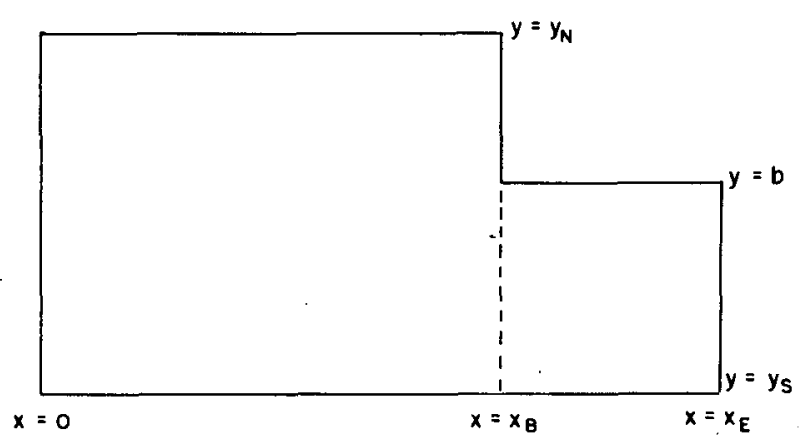

FIG. 1. The model basin geometry. there is $\left[\hat{u}_{r}(y), \hat{h}_{r}(y)\right]$. There may be a reflected Kelvin wave east of $x_{B}$; denote its amplitude by $T^{r}$. West of $x_{B}$ there can only be Rossby waves. North of $b$ at $x_{B}$ there is a wall so $u=0$ and geostrophy then requires that $h$ be independent of $y$. Hence there the transmitted solution $\left(u_{r}, h_{r}\right)$ must be:

$$
u_{r}=0, \quad h_{r}=D^{r}, \quad y \geqslant b,
$$

$D^{r}$ a constant. South of $b$ continuity of $u$ and $h$ across $x=x_{B}$ yields

$$
\begin{array}{ll}
u_{r}=\hat{u}_{r}(y)+T^{r} \psi_{-1}(y), & y<b, \\
h_{r}=\hat{h}_{r}(y)+T^{r} \psi_{-1}(y), & y<b .
\end{array}
$$

DCP shows that

$$
\begin{gathered}
D^{r}=\hat{h}_{r}(b)+T^{r} \psi_{-1}(b) \\
T^{r}=\frac{-\hat{h}_{r}(b) \int_{b}^{y_{N}} \psi_{-1} d y}{2 \int_{y_{S}}^{b} \psi_{-1}^{2} d y+\psi_{-1}(b) \int_{b}^{y_{N}} \psi_{-1} d y} .
\end{gathered}
$$

Note that the only feature of the incident motion that $T^{r}$ and $D^{r}$ depend on is its height at the wall, $\hat{h}_{r}(b)$, and their dependence on this variable is linear. Figure 2 of DCP shows the behavior of $T^{K}, D^{K}, T^{r}$, $D^{r}$ as functions of $b$ for a meridionally infinite basin.

The westward moving solutions include the $n=0$ Rossby mode (cf. CSIII), which cannot exist away from the boundary and so, unlike the other Rossby modes, cannot travel past the corner into the interior. The mass flux associated with this mode must be transferred past the corner and eventually into the large basin. However, the speed of propagation of the Rossby modes west of the corner is slower than that of the $n=0$ mode so that the flux of mass would decrease if the mass density (i.e., height) did not rise in compensation. Most of this rise takes place along the wall north of $y=b$, making up the missing mass flux as the Rossby modes move away from the wall. This is the beta-plane low-frequency version of a coastal Kelvin wave turning the corner. This mass balance can be shown analytically when the corner at $b$ is sufficiently far away from the equator. From (2.5) we may derive a single equation for $h$ :

$$
h_{y y t}-\frac{2 h_{y t}}{y}-y^{2} h_{t}-h_{x}=0 .
$$

If $b \gg 1$, then $y \gg 1$ along the wall, while $h$ is a smooth function of $y$ (i.e., $h_{y} \leqslant \mathrm{O}(1)$ ). Hence we have approximately (cf. Meyers, 1979)

$$
y^{2} h_{t}+h_{x}=0 \text {. }
$$

If the height, $h_{0}=$ constant, is given along the boundary, the solution is $h=h_{0} H\left(x-x_{B}+t / y^{2}\right)$, where $H$ is the Heaviside function. After a time $t$ the total mass north of $y=b$ and west of $x=x_{B}$ is given by 


$$
\begin{aligned}
M_{r} & =\int_{b}^{\infty} \int_{-\infty}^{x_{B}} h_{0} H\left(x-x_{B}+\frac{t}{y^{2}}\right) d x d y \\
& =\int_{b}^{\infty} h_{0} \frac{t}{y^{2}} d y=h_{0} \frac{t}{b} .
\end{aligned}
$$

The form of the $n=0$ wave is approximately $h$ $=-u=A^{\prime} e^{+y^{2} / 2} ;$ a change of variable to $\eta=b-y$ gives

$$
u=A^{\prime} \exp \left[\frac{b^{2}}{2}-b \eta+\frac{\eta^{2}}{2}\right]=A e^{-b \eta} e^{\eta^{2} / 2} .
$$

For $\eta$ small, the second exponential is close to one and this looks like the equation for a coastal Kelvin wave on an $f$-plane. The mass $M_{0}$, arriving at the boundary due to this wave, can be calculated by integrating the zonal mass flux in $y$ and $t$. Expanding the second exponential in its Taylor series and integrating in $\eta$ and $t$ gives

$$
M_{0}=A \frac{t}{b}+A \frac{t}{b^{3}}+\mathrm{O}\left(\frac{1}{b^{5}}\right) .
$$

Since $h$ is continuous at the corner [cf. Eq. (3.5)], $A$ $=h_{0}$ and $M_{r}=M_{0}$ to $\mathrm{O}\left(b^{-3}\right)$. The coastal $n=0$ wave can be thought of as turning the corner and transferring its mass flux into the Rossby modes, producing the constant height $h_{0}$ as it travels up the wall.

\section{Numerical methods}

\section{a. Numerical scheme}

As noted in Section 2, of all the free solutions to (2.5) only the equatorial Kelvin modes propagate eastward. Thus if we write the total solution as in (2.10), i.e.,

$$
(u, v, h)=a_{K}(x, t)\left(\psi_{-1}, 0, \psi_{-1}\right)+\left(u^{\prime}, v^{\prime}, h^{\prime}\right),
$$

then the primed part of the solution, consisting of long Rossby and anti-Kelvin modes, propagates only to the west. The equation governing $a_{K}$ is found by projecting the Kelvin mode onto (2.5):

$$
\frac{\partial a_{k}}{\partial t}+\frac{\partial a_{K}}{\partial x}=f_{K}(x, t)
$$

where

$$
\begin{aligned}
f_{K} & =\frac{(F, G, Q) \cdot\left(\psi_{-1}, 0, \psi_{-1}\right)}{\left(\psi_{-1}, 0, \psi_{-1}\right) \cdot\left(\psi_{-1}, 0, \psi_{-1}\right)} \\
& =\frac{1}{2} \int_{y_{S}}^{y_{N}}(F+Q) \psi_{-1} d y .
\end{aligned}
$$

The appropriate boundary condition for (4.1) is (2.12) at $x=0$. The initial condition is $a_{K}=0$. The solution may be written

$$
\begin{aligned}
a_{K}\left(x, t_{0}+\Delta t\right)=a_{K} & \left(x-\Delta t, t_{0}\right) \\
& +\int_{0}^{\Delta t} f_{K}\left(x-t^{\prime}, t_{0}+t^{\prime}\right) d t^{\prime} .
\end{aligned}
$$

The finite difference approximation to (4.2) is straightforward. Define $\alpha=\Delta t / \Delta x$ and let $p$ $=$ integral part of $\alpha$ and $\gamma=\alpha-p$. Then

$$
\begin{aligned}
a_{K}^{n}(i)=a_{K}^{n-1}(i-\alpha)+ & \Delta x\left\{\gamma f_{K}^{n-1 / 2}\left(i-p-\frac{1}{2}\right)\right. \\
& \left.+\sum_{m=1}^{p} f_{K}^{n-1 / 2}\left(i-m+\frac{1}{2}\right)\right\}
\end{aligned}
$$

where the expressions in parentheses index the longitudes. If $\alpha$ is nonintegral, then $x_{i}-\alpha \Delta x$ is not a grid point and $a_{K}(i-\alpha)$ is found by linear interpolation. The low-order approximation to the integral of $f_{K}$ used in (4.3) is sufficiently accurate as long as $f_{K}$ varies little on the grid scales, $\Delta x$ and $\Delta t$.

A slightly different procedure is needed when $x_{i}$ $-\alpha \Delta x$ lies west of the western boundary $x_{1}$. In this case the characteristic passing through $x_{i}$ at time step $n$ left $x_{1}$ at time level $n-(i-1) / \alpha$, which is after time step $n-1$. We find the value of $a_{K}\left(x_{1}\right)$ at that time by linearly interpolating in time between the values at time steps $n-1$ and $n$. The value of $a_{K}^{n}\left(x_{1}\right)$ is determined from the obvious finite difference version of the boundary condition (2.12) once we have calculated the rest of the solution (i.e., the Rossby and anti-Kelvin waves-the westward propagating part).

The differential equations governing the remainder of the solution are found by subtracting the Kelvin wave equation from (2.5):

$$
\left.\begin{array}{rl}
u_{t}^{\prime}-y v^{\prime}+h_{x}^{\prime} & =F^{\prime}=F-f_{K} \psi_{-1} \\
y u^{\prime}+h_{y}^{\prime} & =G \\
h_{t}^{\prime}+u_{x}^{\prime}+v_{y}^{\prime} & =Q=Q-f_{K} \psi_{-1}
\end{array}\right\} .
$$

The initial conditions for (4.4) are given in (2.8). Boundary conditions cannot be given at the west; instead, as noted above, $u^{\prime}$ at the west determines the initial Kelvin wave amplitude. Equation (2.7) applies north and south while (2.13) determines the eastern values in terms of the Kelvin wave amplitude.

The finite difference versions of (4.4) are defined on the staggered grid depicted in Fig. 2. With $n, i, j$ indexing time, longitude and latitude, respectively, the equations of motion take the form

$$
\begin{aligned}
\frac{1}{2 \Delta t}\left(u_{i, j}^{n}+u_{i+1, j}^{n}-u_{i, j}^{n-1}-u_{i+1, j}^{n-1}\right)-\frac{1}{2} y_{j}\left(v_{i+1 / 2, j+1 / 2}^{n-1 / 2}+v_{i+1 / 2, j-1 / 2}^{n-1 / 2}\right) & \\
& +\frac{1}{2 \Delta x}\left(h_{i+1, j}^{n-1}+h_{i+1, j}^{n}-h_{i, j}^{n-1}-h_{i, j}^{n}\right)=F_{i+1 / 2, j}^{n-1 / 2,}
\end{aligned}
$$




$$
\begin{gathered}
\frac{1}{2}\left(y_{j} u_{i, j}^{n}+y_{j+1} u_{i, j+1}^{n}\right)+\frac{1}{\Delta y}\left(h_{i, j+1}^{n}-h_{i, j}^{n}\right)=G_{i, j+1 / 2}^{n}, \\
\frac{1}{2 \Delta t}\left(h_{i, j}^{n}+h_{i+1, j}^{n}-h_{i, j}^{n-1}-h_{i+1, j}^{n-1}\right)+\frac{1}{2 \Delta x}\left(u_{i+1, j}^{n-1}+u_{i+1, j}^{n}-u_{i, j}^{n-1}-u_{i, j}^{n}\right) \\
+\frac{1}{\Delta y}\left(v_{i+1 / 2, j+1 / 2}^{n-1 / 2}-v_{i+1 / 2, j-1 / 2}^{n-1 / 2}\right)=Q_{i+1 / 2, j}^{n-1 / 2}
\end{gathered}
$$

We march the equations forward in time and from east to west in space; that is, in the direction of wave propagation. To describe the procedure, we assume that all variables are known at the previous time level $(n-1)$ and the grid longitude immediately to the east $(i+1)$. Our solution method is analogous to the usual procedure for finding equatorial modes (e.g., Moore and Philander, 1977). We first solve for the unknowns $u_{i, j}^{n}$ and $h_{i, j}^{n}$ in terms of known quantities and the unknowns $v_{j+1 / 2}, v_{j-1 / 2}$ (the time index $n$ $-1 / 2$ and longitude index $i+1 / 2$ are omitted):

$$
\begin{aligned}
u_{i, j}^{n}=\frac{\Delta t}{1-\alpha^{2}}\left[R_{j}^{1}+v_{j-1 / 2}\left(y_{j}+\frac{2 \alpha}{\Delta y}\right)\right. & \\
& \left.+v_{j+1 / 2}\left(y_{j}-\frac{2 \alpha}{\Delta y}\right)\right],
\end{aligned}
$$

where

$$
\begin{aligned}
R_{j}^{1}= & 2\left(F_{j}+\alpha Q_{j}\right)+\frac{\left(1-\alpha^{2}\right)}{\Delta t} u_{i+1, j}^{n-1} \\
& -\frac{\left(1+\alpha^{2}\right)}{\Delta t}\left(u_{i+1, j}^{n}-u_{i, j}^{n-1}\right)-\frac{2 \alpha}{\Delta t}\left(h_{i+1, j}^{n}-h_{i, j}^{n-1}\right) ; \\
h_{i, j}^{n}= & \frac{\Delta t}{1-\alpha^{2}}\left[R_{j}^{3}+v_{j-1 / 2}\left(\frac{2}{\Delta y}+\alpha y_{j}\right)\right. \\
& \left.+v_{j+1 / 2}\left(\frac{-2}{\Delta y}+\alpha y_{j}\right)\right], \quad \text { (4.6b) }
\end{aligned}
$$

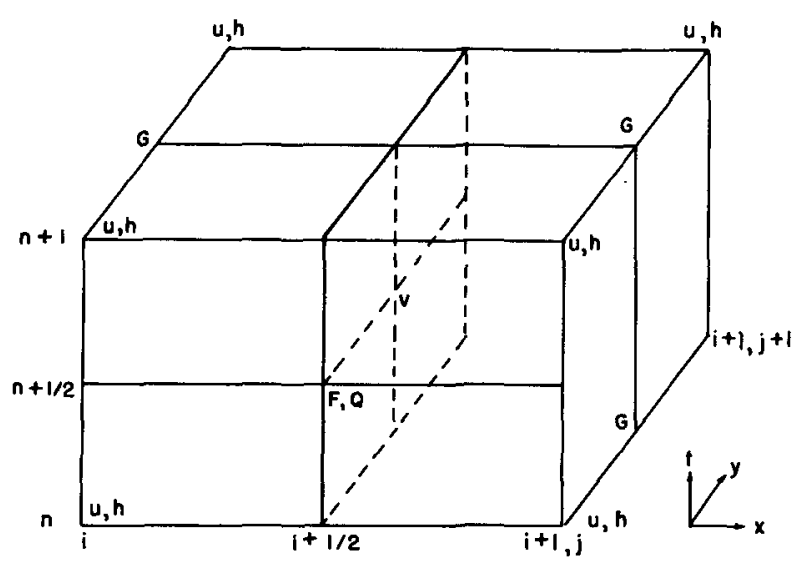

FIG. 2. Finite difference grid, staggered in space and time. where

$$
\begin{aligned}
R_{j}^{3}=2( & \left.Q_{j}+\alpha F_{j}\right)+\frac{\left(1-\alpha^{2}\right)}{\Delta t} h_{i+1, j}^{n-1} \\
& -\frac{\left(1-\alpha^{2}\right)}{\Delta t}\left(h_{i+1, j}^{n}-h_{i, j}^{n-1}\right)-\frac{2 \alpha}{\Delta t}\left(u_{i+1, j}^{n}-u_{i, j}^{n-1}\right)
\end{aligned}
$$

and $\alpha=\Delta t / \Delta x$ represents an inverse numerical phase speed. An equation in $v$ only can be found by substituting expressions $(4.6 \mathrm{a}, \mathrm{b})$ in $(4.5 \mathrm{~b})$ : The result is

$$
\begin{aligned}
\hat{L} v_{j+1 / 2}=\frac{2\left(1-\alpha^{2}\right)}{\Delta t} G_{i, j+1 / 2}^{n} & \\
& \quad-\left(y_{j} R_{j}^{1}+y_{j+1} R_{j+1}^{1}\right)-\frac{2}{\Delta y}\left(R_{j+1}^{3}-R_{j}^{3}\right),
\end{aligned}
$$

where the operator $\hat{L}$ is defined by

$$
\begin{aligned}
\hat{L} v_{j+1 / 2} & =v_{j-1 / 2}\left(y_{j}^{2}-\frac{4}{\Delta y^{2}}\right)+v_{j+1 / 2} \\
& \times\left(y_{j}^{2}+y_{j+1}^{2}+\frac{8}{\Delta y^{2}}+4 \alpha\right)+v_{j+3 / 2}\left(y_{j+1}^{2}-\frac{8}{\Delta y^{2}}\right) .
\end{aligned}
$$

Note that $\hat{L} / 4$ is the finite difference analogue to the operator $\tilde{L} \equiv \partial^{2} / \partial y^{2}-y^{2}$. Given the value of $\left(u_{N X, j}^{n}\right.$, $\left.h_{N X, j}^{n}\right)$ at the eastern boundary, the tridiagonal system (4.7) can be solved for $v$ at $i=N X-1 / 2$ with the boundary condition $v=0$ at the northern and southern boundaries. Equations $(4.6 \mathrm{a}, \mathrm{b})$ then give $u$ and $h$ at $N X-1$, the procedure having moved the solution over to the left by one grid point. The scheme then continues calculating successive values of $u$ and $h$ to the left until the western boundary is reached.

Initial conditions $(n=0)$ are determined by the finite difference analogues of (2.8). Values of $u^{n}$ and $h^{n}$ at the eastern boundary $(i=N X)$ are found from the finite difference analogue of (2.13); the requisite value of $a_{K}^{n}\left(x_{N X}\right)$ is obtained from (4.3). The only tricky point in using (2.13) is that the Kelvin mode meridional structure function $\psi_{j}$ must be the exact solution of the unforced version of $(4.5 \mathrm{~b})$ with $u$ $=h$; that is

$$
\frac{1}{2}\left(y_{j} \psi_{j}+y_{j+1} \psi_{j+1}\right)+\frac{1}{\Delta y}\left(\psi_{j+1}-\psi_{j}\right)=0 .
$$


When the Gulf of Guinea is included in the basin, the boundary conditions and transmission coefficients are calculated using the finite difference forms of the appropriate equations given in Section 3 (see Patton, 1981, for details).

\section{b. Accuracy and stability}

The accuracy and stability of this scheme is described in detail in Patton (1981). The most salient points will be summarized here. The scheme (4.5) is second order accurate in time and space. The Kelvin wave procedure (4.3) is exact for integral values of $\alpha$; otherwise a second order interpolation is needed to find $a_{K}$ at $i-\alpha$. In the absence of forcing and damping, both the finite difference equation for the Kelvin wave (4.3) and those for all other modes (4.5) identically conserve mass and energy. They do so in both time and space, i.e., they are fully conservative and not just semi-conservative. There is some loss (or gain) at the boundaries, where reflections exchange mass and energy between the Kelvin and Rossby waves. It is possible to formulate consistent numerical boundary conditions that would be mass conserving, but since in practice the ones we use generate very small changes in mass and energy it was not deemed necessary.

Another view of the accuracy of our scheme is afforded by considering how well it models the westward propagation of a meridional mode with phase speed $C_{m}$. The expression for the error,

$$
\epsilon=-\frac{1}{12} \frac{\partial^{3} u}{\partial t^{3}} \Delta t^{3}\left[1-\left(\alpha C_{m}\right)^{-2}\right],
$$

shows the importance of the parameter $p=\alpha C_{m}$, the number of grid spaces the wave moves in one time step. If $p=-1$, then the numerical speed matches the mode speed and the model moves the wave exactly along the characteristic without error. For a wave that crosses many grid boxes in a time step $(|p|$ $\gg 1$ ) the expression in square brackets is close to one. Then, for example, if we use a 10-day time step, the relative error in motions with a 2 -month period is about $10 \%$, reducing to about $3 \%$ and $1 \%$ for 3 month and 4-month periods, respectively. As $p$ decreases, the error decreases until $p=-1$ and then begins to rise again (in absolute value), attaining the same value as for large $p$ when $p \sim-2^{-1 / 2}$. With $\Delta t$ $=10$ days, $\Delta x=1$ degree of longitude, a second baroclinic mode equivalent depth $(\sim 20 \mathrm{~cm})$ and $C_{m}$ $\sim(2 m+1)^{-1}$ this occurs when $m \sim 10$. We conclude that a 10-day time step with a one-degree grid spacing will yield acceptable accuracy in simulations of the annual cycle.

Our scheme is unconditionally stable for westwardtraveling waves. However, it is unstable for eastwardpropagating modes and there is a single eastward mode allowed by the system (4.4): the equatorial
Kelvin wave. Therefore, although the direct forcing for a Kelvin wave has been removed from (4.4), an unstable Kelvin wave will be generated from truncation error and other noise as the scheme marches westward. However, since we know that this computational error must have precisely the Kelvin wave form [i.e., $u=h=a_{K} \psi_{-1}$, with $\psi_{-1}$ given by (4.8)] it is a simple matter to filter it from the solution. This is now done efficiently as a part of the calculation of $f_{K}$.

\section{Effects of basin geometry}

In this section and the next we consider the model response to periodic forcings. This work extends the analytic calculations of CSIV to (i) the more complex geometry of a realistic Gulf of Guinea and (ii) a spatially varying wind amplitude. In our first set of calculations we used a model Atlantic basin nondimensionalized as for a first baroclinic mode forced by a zonal wind of the form $F=\exp \left(-0.1 y^{2}\right) \cos \omega t$, where $\omega$ is the annual frequency. This corresponds to the case shown in CSIV in their Fig. 5. In addition to changes in the geometry and the method of solution, the present calculation differs by including Rayleigh friction (nondimensionally, $r=0.01$; the frequency $\omega=0.026$ ). While the model will run with zero friction, it is desirable to damp the poorly resolved, slowly moving short (zonal and meridional scale) waves generated at the eastern side as the reflection of Kelvin waves.

Figure 3 shows fields of $u$ and $h$ at $\omega t=0$ (January) when the forcing is a maximum; Figure 4 displays them at $\omega t=\pi / 2$ (April) when the forcing is zero. The annual variation of the phase and amplitude of $h$ along the equator is shown in Fig. 5. These pictures are similar to the results shown in CSIV (Fig. 5) in many ways. Along the equator, the height is either in phase with the wind or $180^{\circ}$ out of phase with it, except in a narrow region where the amplitude is a minimum and the phase changes abruptly. As in CSIV, the minimum is not quite zero: there is no true node in the solution. In our bounded basin this near-nodal pivot point is closer to the center of the basin than is true for the meridionally infinite basin of CSIV. As one moves poleward the response is no longer in phase with the wind, as is readily apparent in Fig. 4, a time when the wind is zero. The feature in the southeast corner of Fig. 4a (absent in Fig. 3a) bears a qualitative resemblance to the Angola dome (e.g., Mazieka, 1967). The nonlocal influence of the altered geometry is slight.

The principal effect of the coast at $5^{\circ} \mathrm{N}$ is to add a narrow boundary layer, as is evident from Fig. 6, which shows the variation of $h$ at $2^{\circ} \mathrm{E}$. (It also can be seen clearly in Fig. 3b.) Note that the amplitude is greatest at the coast and near the equator, diminishing in between. There is little if any apparent 


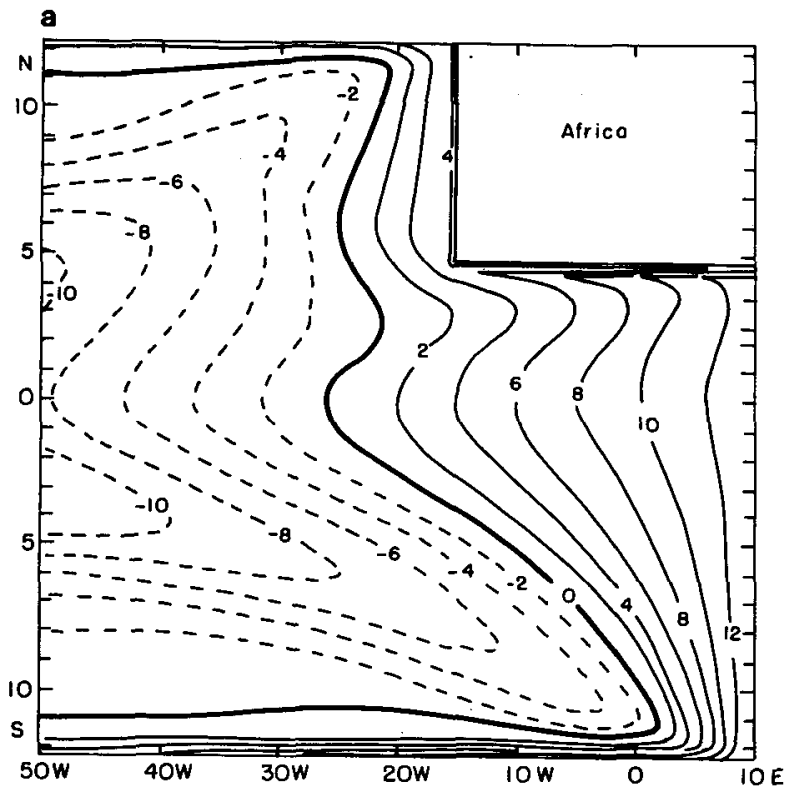

is a standing, periodic pattern, comprised of many waves. The lack of phase variations results from interference among these waves.

The narrowness of the boundary layer and the absence of any obvious influence of the boundary on the interior recommend an analysis of our results in terms of (i) an interior, forced solution plus (ii) a boundary layer of unforced motions generated in order to satisfy the boundary condition $v=0$ at the Guinea coast. The boundary layer visible in our solution is too narrow to be a single coastally trapped meridional mode: CSIII show that the appropriate
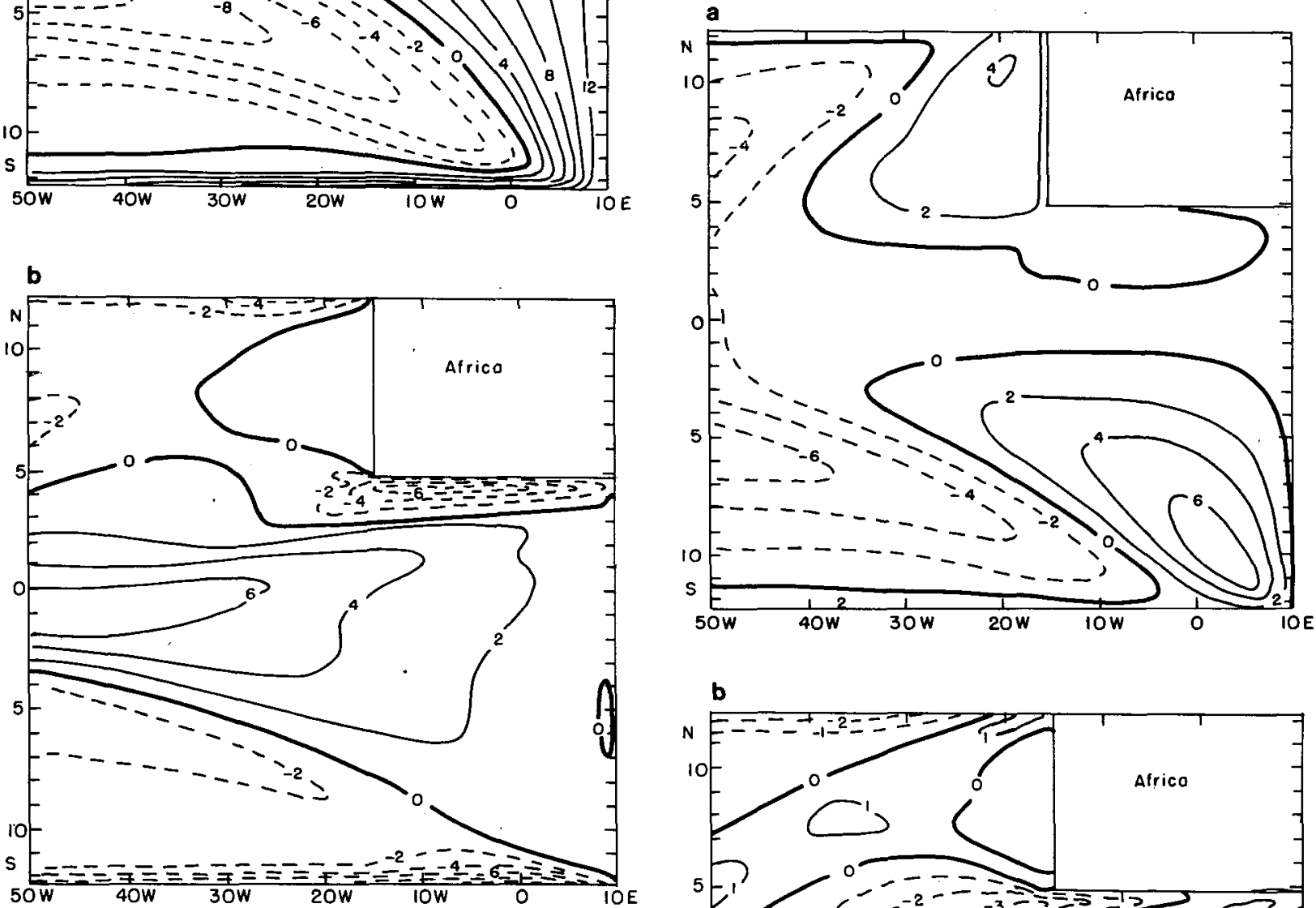

FIG. 3. Response in the model Atlantic basin for uniform periodic forcing $F=\exp \left(0.1 y^{2}\right) \cos \omega t$ when $\omega t=0$ (January): (a) height contours $h$; (b) zonal velocity $u$.

phase difference between the equator and the coast. The variation of $h$ along the coast in time and longitude (Fig. 7) shows that there is only a slight westward phase propagation along the coast (cf. CSIV). This is in fact consistent with data, although the data has sometimes been interpreted differently [see Merle et al. (1980); Picaut (1983)]. A single coastal wave generated in response to an impulsive forcing would generate phase differences consistent with its phase speed (of about $50-100 \mathrm{~km} \mathrm{day}^{-1}$ ). The response shown in Fig. 7 (and, arguably, the Atlantic Ocean)

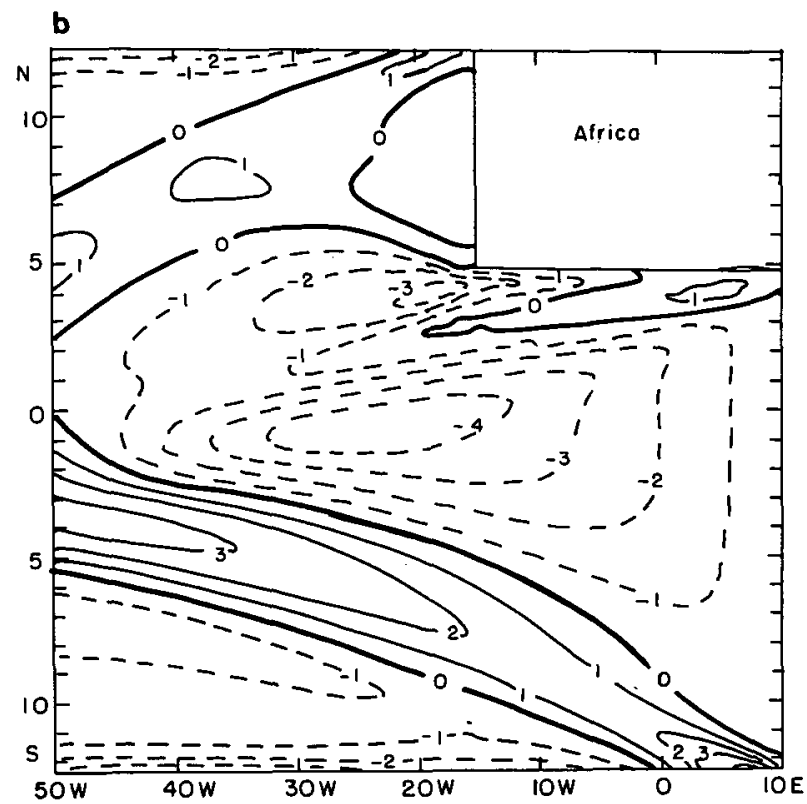

Fig. 4. As in Fig. 3 but for $\omega t=\pi / 2$ (April):

(a) height contours $h$; (b) zonal velocity $u$. 


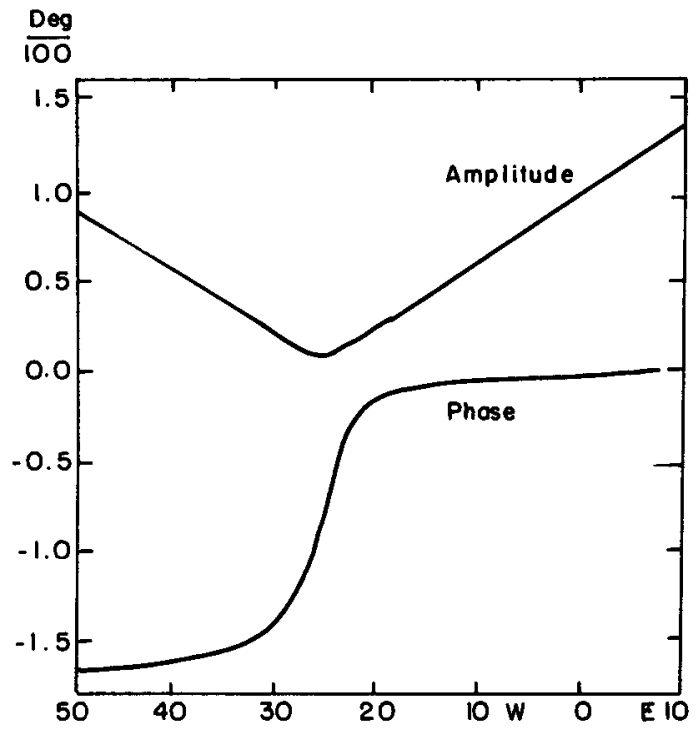

FiG. 5. Amplitude and phase of height along the equator for the uniform periodic forcing case. Phase is shown in units of degrees/ 100 and is relative to $t=0$ when the phase of the wind is zero.

mode is the $n=0$ Rossby mode and from their Fig. 4 it clearly extends to the equator. Rather, it must be a sum of many westward-moving Rossby waves. Each of these, and hence their sum, satisfies (2.5) with the forcings set to zero. The small scale of the boundary layer suggests that $\partial / \partial y>y$ and introduces the scaling

$$
\begin{gathered}
v=\mathrm{O}(1), \quad h=\mathrm{O}(1), \quad u=\mathrm{O}\left(\omega^{-1 / 2}\right), \\
\frac{\partial}{\partial y}=\mathrm{O}\left(\omega^{-1 / 2}\right), \quad y=\mathrm{O}(1), \quad \frac{\partial}{\partial x}=\mathrm{O}(1),
\end{gathered}
$$

where $\partial / \partial t \approx \omega \ll 1$. (See Patton, 1981, for details.) The continuity equation $(2.5 \mathrm{c})$ then shows that to

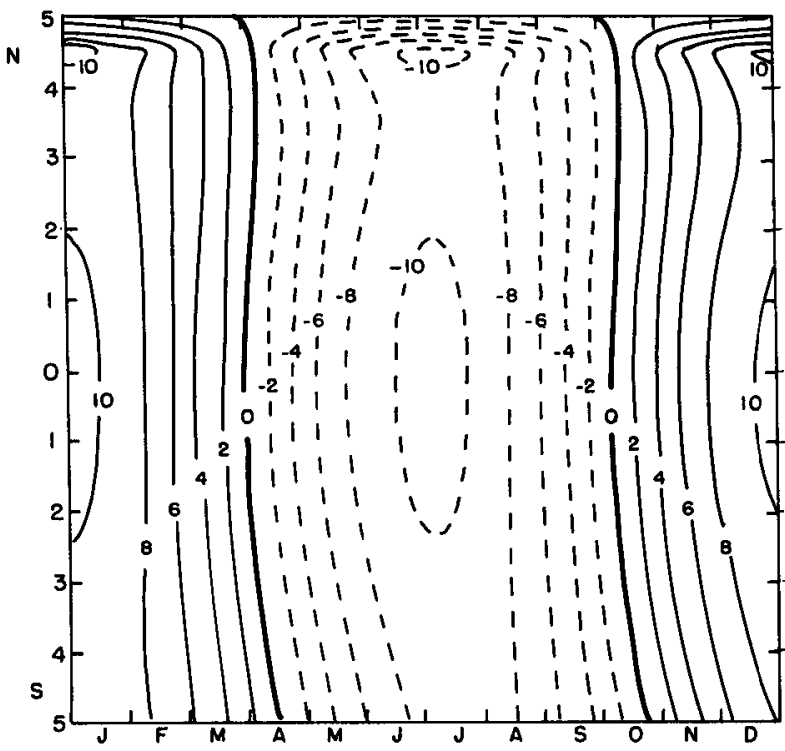

FIG. 6. Height as a function of time and latitude at $2^{\circ} \mathrm{E}$.

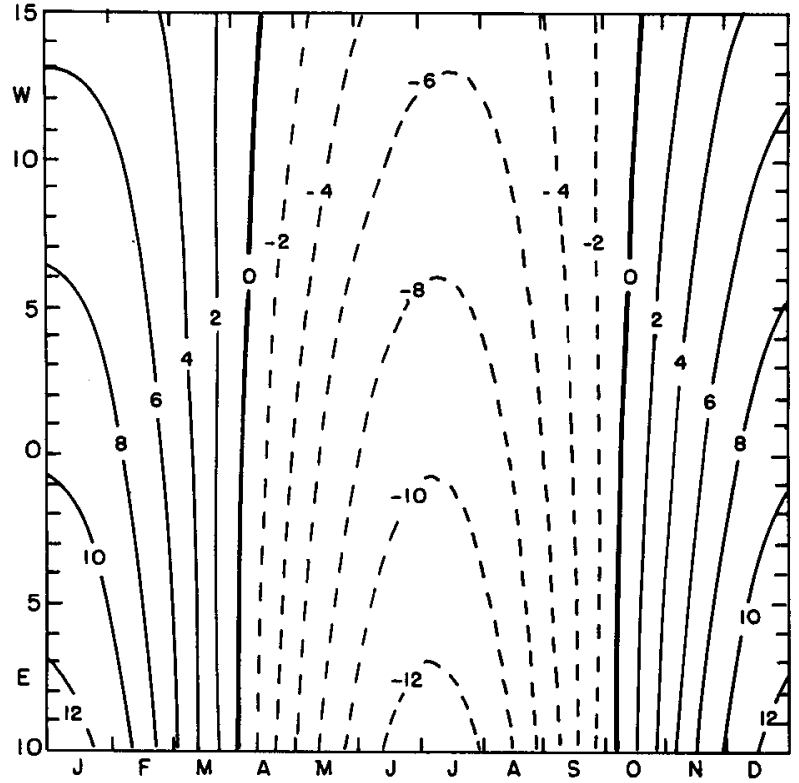

Fig. 7. Height as a function of time and longitude along the model Guinea coast at $5^{\circ} \mathrm{N}$.

$O\left(\omega^{3 / 2}\right)$ we can define a streamfunction $\psi^{B}$ for the boundary layer velocities $u^{B}$ and $v^{B}$ :

$$
u^{B}=-\psi_{y}^{B} ; \quad v^{B}=\psi_{x}^{B} .
$$

Consistent with the scaling (5.1) the lowest-order vorticity equation derivable from $(2.5)$ is

$$
\frac{\partial^{2}}{\partial y^{2}} \psi_{t}^{B}+\psi_{x}^{B}=0
$$

The largest term neglected, $y^{2} \psi_{t}^{B}$, enters at $O(\omega)$ : at $\mathrm{O}\left(\omega^{3 / 2}\right)$ the streamfunction relation (5.2) breaks down. Denoting the interior meridional velocity by $v^{I}$, the boundary conditions are $\psi^{B}=0$ at the eastern boundary, $x_{E}$ and

$$
\psi^{B}(x, b)=-\int_{x_{E}}^{x} v^{I}\left(x^{\prime}, b\right) d x^{\prime} \equiv-\psi^{\prime}(x)
$$

at the coast $y=b$. When Rayleigh friction is included (cf. Sec. 2) it introduces a term similar to the time derivative and $\partial / \partial t$ can be replaced by $i \omega+r=\delta$. By defining $\eta=b-y, \sigma=x_{E}-x$, so that distances are measured positive from the zonal coast and the eastern boundary, the boundary layer problem can be cast in the standard form

$$
\left.\begin{array}{cc}
\psi_{\sigma}^{B}=\delta \psi_{\eta \eta}^{B}, \\
\psi^{B}=-\psi^{\prime}(\sigma), & \eta=0 \\
\psi^{B} \rightarrow 0, & \eta \rightarrow \infty \\
\psi^{B}=0, & \eta=0
\end{array}\right\} .
$$

Even before solving, it is evident from the boundary condition (5.3) that the phase variation (and hence phase speed) along the coast will be determined by the interior solution. This interior solution, which is essentially the same as the meridionally unbounded 
solution of CSIV, exhibits very little phase variation, in contrast to a coastal wave.

When $\delta$ is real, (5.2) is a diffusion equation with $\sigma$ the time-like variable and $\eta$ the space-like one. One expects that the boundary layer would broaden as one moves westward from the eastern corner (cf. Cane and DuPenhoat, 1982, Section 4); our solution (viz. Fig. 4b) does have this character. If $\delta$ is pure imaginary then the solution would oscillate with an ever-broadening wavelength in $y$ as one proceeds westward. Our parameters give both a damping and an oscillatory effect, with the former the more readily visible. Eventually the boundary layer becomes wide enough so that the assumption that $\partial / \partial y>y$ is no longer valid. At this point the term $-\delta y^{2} \psi^{B}$ enters on the right-hand side of (5.2) and the boundary layer width no longer increases downstream (westward). For the first baroclinic mode this occurs at a point $3600 \mathrm{~km}$ from the eastern boundary-outside the Gulf of Guinea.

\section{Effect of non-uniform amplitude in the wind forcing}

As we have seen, even the simplest periodic forcing gives rise to considerable phase and amplitude variation in the ocean. The reflection and interference of the long, low frequency equatorial waves create a response which may have little resemblance to the propagation of a single wave or to local forcing. The increased complexity of the real winds also raises the issue of how the ocean redistributes variations in the phase and amplitude of the wind. The equilibrium adjustment of the height along the equator, for instance, would have to change from a simple "seesaw" to more complicated undulations to respond to a shifting phase in the forcing.

The seasonal signal for the zonal wind field in the equatorial Atlantic is much larger in the western half of the basin than in the east (Hastenrath and Lamb, 1977). Moore et al. (1978) and Adamec and O'Brien (1978) considered only the winds in the western Atlantic in their studies of upwelling in the Gulf of Guinea. Our model periodic winds were modified so that the zonal forcing was nonzero in the western $3 / 10$ of the basin only. Figure 8 shows the amplitude and phase of the height along the equator for this case. Compared with Fig. 5 for a uniform amplitude, the difference is a shifting of the approximate node of the oscillation to near the center of the forcing. To the east of this, the ocean responds in phase with the wind. The far western part of the basin is $180^{\circ}$ out-of-phase, as would be expected. The change of the response from in-phase to out-of-phase takes place over roughly the same distance as in the uniform forcing case. The amplitude over the eastern part of the basin is almost uniform and is diminished compared to the uniform wind forcing case where the amplitude increased toward the east. The reason for the constant amplitude, constant phase region to the east of the forcing may be understood by considering the form of the analytic solution (cf. CSIV). Of the

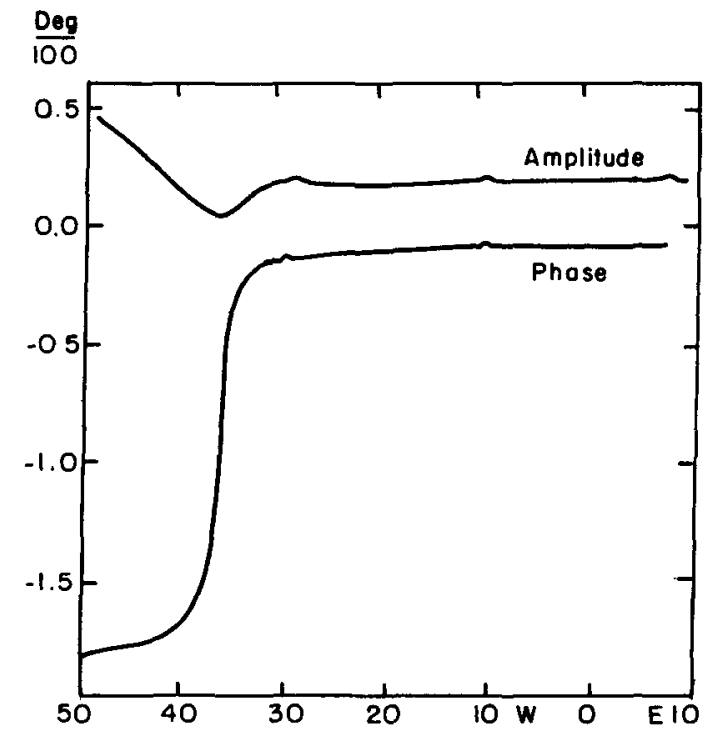

FIG. 8. Amplitude and phase of height along the equator for periodic winds nonzero only over the western $30 \%$ of the basin $\left(50^{\circ} \mathrm{W}\right.$ to $\left.32^{\circ} \mathrm{W}\right)$. Cf. Fig. 5 .

modes of motion that are directly forced only the equatorial Kelvin wave propagates eastward. Therefore the periodic response east of the forcing region consists of a Kelvin wave plus the Rossby waves generated when it is reflected off the eastern boundary. In a meridionally infinite basin the height along the equator due to the Kelvin wave and its reflections takes the form (Cane and Moore, 1981)

$$
h \propto\left[\cos 2 \omega\left(x-x_{E}\right)\right]^{1 / 2} e^{i \omega t} .
$$

The discussion of the effects of the Guinea coast in the last section indicate its form will be little modified by meridional boundaries. [The small "bumps" in Fig. 9 appear to be due to the Guinea coast.] The phase of $h$ relative to the wind will not change from zero until $\omega\left(x_{E}-x\right)=\pi / 4$, which lies outside the basin for the present parameter values. The amplitude is approximately $1-\left[\omega\left(x-x_{E}\right)\right]^{2}$, which varies by a barely perceptible $15 \%$ east of the forcing. In the previous section we saw that there was little phase variation along the Guinea coast for a spatially uniform wind. If the wind is confined to the west the phase along the coast becomes even more uniform.

\section{Discussion}

The principal result presented in this paper is a fast, efficient numerical procedure for modeling the linear, low-frequency motions on an equatorial beta plane. The model is particularly well suited to studying seasonal and interannual variability. The size of the time step is limited only by accuracy considerations, notably the need to resolve the variations in the wind forcing. In practice, we find that a 10-day time step provides sufficient accuracy in simulations of seasonal response. (This gives 6 points per wavelength for motions with a 2-month period; also see the error analysis in Section $4 \mathrm{~b}$.) 
Our numerical method makes use of the special characteristics of low-frequency equatorial dynamics. We extract the Kelvin mode part from the entire response and solve for it by integrating along characteristics; in essence, an analytic procedure. The remainder of the solution (Rossby modes and a coastal, Anti-Kelvin mode) propagates only westward. We use this fact to design an implicit scheme that achieves computational efficiency by marching westward longitude-line by longitude-line. Formulation of the model equations is straightforward, but proper formulation of computationally tractable boundary conditions is not. To do so we rely on the methodology developed in the series of papers by Cane and Sarachik (especially CSII and CSIII).

One of the objectives of our modeling effort was to handle realistic basin geometry, not just simple rectangles. To this end a set of formulas for the influence of partial boundaries on low-frequency equatorial waves was developed. The complete derivation is given in DuPenhoat et al. (1983); in this paper we review the results for a partial boundary like the western end of the Gulf of Guinea. The amplitude of a Kelvin wave increases as it passes such a corner, though only by a few percent for a coast as far from the equator as the Guinea coast. Even this small effect is important in long term integrations; its omission resulted in a substantial loss of mass in earlier calculations.

The application of our model considered here is an extension to a basin geometry that models the Gulf of Guinea of the results of CSIV on the response to periodic winds in a meridionally unbounded ocean. We first consider a zonally uniform forcing, a case identical to one treated in CSIV. We find only minor differences from their results away from the Guinea coast. Along the coast there is a narrow coastal current, too narrow to be a single wave; instead, it is a sum of many waves. There is little, if any, phase variation along the coast and very little phase lag between the equator and the coast. We analyze the boundary layer using standard boundary layer methods and show that the phase of the coastal regime is determined by the interior solution; so the lack of phase variation is to be expected in this periodic problem.

Next we consider annual winds in the western equatorial Atlantic only. This is the periodic version of the wind forcing used in earlier initial value studies of Gulf of Guinea upwelling (Moore et al., 1978; Adamec and O'Brien, 1978). Phase variation along the coast is even less than before: there is essentially no phase or amplitude variation anywhere to the east of the forcing region. This result may be readily explained by existing theory for the response to periodic winds.

We conclude that the Guinea current system, including the annual upwelling, is better described as a periodic response rather than an initial value one.
The lack of westward propagation along the coast and the narrow meridional scale of this boundary layer are both signatures of a response to periodic forcing.

Acknowledgments. We are grateful to Prof. Moshe Israeli for much useful guidance in the construction of the numerical scheme. This work was supported by NASA Grant NGR 22-009-727 and NSF Grant OCE 79-22046 at M.I.T.

\section{REFERENCES}

Adamec, D., and J. J. O'Brien, 1978: The seasonal upwelling in the Gulf of Guinea due to remote forcing. J. Phys. Oceanogr., 8, 1050-1060.

Busalacchi, A. J., and J. J. O'Brien, 1980: The seasonal variability in a model of the tropical Pacific. J. Phys. Oceanogr., 10, 1929-1951.

, and - 1981: Interannual variability of the equatorial Pacific in the 1960s. J. Geophys. Res., 86, 10901-10907.

Cane, M. A., 1984: Modeling sea level during El Niño. J. Phys. Oceanogr., 14, 1864-1874.

, and E. S. Sarachik, 1976: Forced baroclinic ocean motions. I. The linear equatorial unbounded case. J. Mar. Res., 34, 629-665.

, and - 1977: Forced baroclinic ocean motions. II. The linear equatorial bounded case. J. Mar. Res., 35, 395-432.

, and - 1979: Forced baroclinic ocean motions. III. The linear equatorial basin case. J. Mar. Res., 37, 355-398.

- and D. W. Moore, 1981: A note on low frequency equatorial basin modes. J. Phys. Oceanogr., 11, 1578-1584.

- and E. S. Sarachik, 1981: The response of a linear baroclinic equatorial ocean to periodic forcing. The linear equatorial periodic case. J. Mar. Res., 39, 651-693.

- , and Y. DuPenhoat, 1982: The effects of islands on lowfrequency equatorial motions. J. Mar. Res., 40, 937-962.

- , and E. S. Sarachik, 1983: Equatorial oceanography. Rev. Geophys. and Space Phys. 21, 1137-1148.

DuPenhoat, Y., M. A. Cane, and R. J. Patton, 1983: Reflections of low frequency equatorial waves on partial boundaries, Hydronamics of the Equatorial Ocean, J. C. J. Nihoul, Ed., Elsevier, 237-258.

Hastenrath, S., and P. J. Lamb, 1977: Climate Atlas of the Tropical Atlantic and Eastern Pacific Oceans, University of Wisconsin Press.

Lighthill, M. J., 1969: Dynamic response of the Indian Ocean to onset of the southwest monsoon. Phil. Trans. Roy. Soc., A265, 45-92.

Mazeika, P. A., 1967: Thermal domes in the eastern tropical Atlantic Ocean. Limnol. Oceanogr., 12, 537-539.

Merle, J., M. Fieux and P. Hisard, 1980: Annual signal and interannual anomalies of sea surface temperature in the eastern equatorial Atlantic Ocean. Deep-Sea Res., (Gate Suppl. II) 77-101.

Meyers, G., 1979: On the annual Rossby wave in the tropical North Pacific Ocean. J. Phys. Oceanogr., 9, 663-674.

Moore, D. W., and S. G. H. Philander, 1977: Modeling of the tropical ocean circulation. (Chap. 8), The Sea, Vol. 6, E. D. Goldberg, I. N. Cave, J. J. O'Brien and J. H. Steek, Eds., Interscience, $1048 \mathrm{pp}$.

- P. Hisard, J. McCreary, J. Merle, J. O’Brien, J. Picaut, J. M. Verstraete and C. Wunsch, 1978: Equatorial adjustment in the eastern Atlantic. Geophys. Res. Letters, 5, 637-640.

Patton, R. J., 1981: A numerical model of equatorial waves with application to the seasonal upwelling in the Gulf of Guinea. MS thesis, M.I.T., 120 pp.

Picaut, J., 1983: Propagation of the seasonal upwelling in the eastern equatorial Atlantic. J. Phys. Oceanogr., 13, 18-37. 